Draft version March 21, 2018

Preprint typeset using $\mathrm{IATEX}_{\mathrm{E}}$ style emulateapj v. 12/16/11

\title{
FORMATION OF HYDROXYLAMINE ON DUST GRAINS VIA AMMONIA OXIDATION
}

\author{
Jiao He, Gianfranco Vidali \\ Physics Department, Syracuse University, Syracuse, NY 13244, USA \\ JEAN-LOUis Lemaire \\ Paris Observatory, Paris, France \\ ROBIN T. GARROD \\ Center for Radiophysics and Space Research, Cornell University, Ithaca, NY 14853, USA \\ Draft version March 21, 2018
}

\begin{abstract}
The quest to detect prebiotic molecules in space, notably amino acids, requires an understanding of the chemistry involving nitrogen atoms. Hydroxylamine $\left(\mathrm{NH}_{2} \mathrm{OH}\right)$ is considered a precursor to the amino acid glycine. Although not yet detected, $\mathrm{NH}_{2} \mathrm{OH}$ is considered a likely target of detection with ALMA. We report on an experimental investigation of the formation of hydroxylamine on an amorphous silicate surface via the oxidation of ammonia. The experimental data are then fed into a simulation of the formation of $\mathrm{NH}_{2} \mathrm{OH}$ in dense cloud conditions. On ices at $14 \mathrm{~K}$ and with a modest activation energy barrier, $\mathrm{NH}_{2} \mathrm{OH}$ is found to be formed with an abundance that never falls below a factor 10 with respect to $\mathrm{NH}_{3}$. Suggestions of conditions for future observations are provided.
\end{abstract}

Keywords: ISM: molecules - ISM: atoms — ISM: abundances — ISM:dust, extinction — Physical

Data and Processes: astrochemistry

\section{INTRODUCTION}

With molecules detected in space increasing in number and growing in complexity, one of the main goals in astronomy today is to find a link between astrochemistry and astrobiology (Wincel et al. 2000). Prebiotic molecules have been already observed that can be considered as precursors of complex organic compounds like amino acids and prebiotic material. N-bearing molecules are necessary precursors of such prebiotic material and among them hydroxylamine $\left(\mathrm{NH}_{2} \mathrm{OH}\right)$ appears to be one of the best candidates. Both experimental and theoretical studies (Blagojevic et al. 2003; Snow et al. 2007) have shown that in its protonated form it can react in the gas phase with acetic and propanoic acids to yield protonated glycine (the simplest non-chiral amino-acid) and protonated alanine (the second simplest and chiral amino-acid). However, the question is still under debate whether such a synthesis happens in the gas phase or by surface reactions. Actually, in a recent work Barrientos et al. (2012) dismiss the possibility that precursors of glycine could be efficiently produced from the reactions of hydroxylamine-derived ions with acetic acid in the gas phase. This is confirmed by recent work (Garrod 2013) using a new three-phase chemical model that fully couples gas-phase, grain-surface and bulk-ice chemistry, which shows that glycine can be formed much more efficiently on grain surfaces than through gas-phase reactions.

Currently, the main problem is the lack of astronomical detection (either in the inter- or circum-stellar media, ISM and CSM) of both hydroxylamine and glycine $\left(\mathrm{NH}_{2} \mathrm{CH}_{2} \mathrm{COOH}\right)$, and this is obviously correlated to their low predicted abundances. Searches for hydrox-

gvidali@syr.edu ylamine have been done by Pulliam et al. (2012) observing seven bright sources toward IRC+10216, Orion KL, Orion S, Sgr B2(N), Sgr B2(OH), W3IRS5, and W51M using the NRAO $12 \mathrm{~m}$ telescope on Kitt Peak, and by Charness (2013) toward L1157-B1 using the CARMA Radio Telescope Array. Glycine of cometary origin has been detected in STARDUST-returned samples (Glavin et al. 2008; Elsila et al. 2009), but it is unknown whether it represents pristine interstellar material. A chemically related species that is a possible direct precursor of glycine, amino acetonitrile $\left(\mathrm{NH}_{2} \mathrm{CH}_{2} \mathrm{CN}\right)$, has been detected (Belloche et al. 2008) in Sgr B2(N). Glycine detection in the ISM or CSM is claimed to be highly plausible using ALMA towards bright nearby sources with narrow emission lines (Garrod 2013). Such sources are luminous high-mass young stellar objects and low-mass protostars, also called hot cores and corinos. They are regions surrounded by dense material $\left(10^{8}\right.$ $\mathrm{cm}^{-3}$ for hot cores and $10^{6} \mathrm{~cm}^{-3}$ for hot corinos) and warm ( $\sim 250 \mathrm{~K}$ and $100 \mathrm{~K}$, respectively) close to the core. This material is much colder farther away. Deep within such dense regions, cosmic-ray (CR) ionized helium, $\mathrm{He}^{+}$, breaks up the very stable $\mathrm{N}_{2}$ molecule, liberating $\mathrm{N}$ atoms that interact with abundant $\mathrm{H}$ atoms to yield $\mathrm{NH}_{3}$, which then sticks to cold grains (Tielens 2005). It is worth noting that the composition of hot-cores resembles that of interstellar ices with high abundances of hydrogenated species as $\mathrm{H}_{2} \mathrm{O}$ and $\mathrm{NH}_{3}$ (Tielens 2005). Hot cores and corinos are in general places where many terrestrial-like organic molecules are observed. More recently, radiative transfer calculations of glycine emission show that low-mass star-forming regions, in their earliest stages of cold pre-stellar cores, may be better regions for the detection of glycine (Jiménez-Serra et al. 2014). Water detection in the low-mass pre-stellar core L1544 
(Caselli et al. 2012) indicates that a fraction of the grain mantles has desorbed into the gas phase. This makes the observation of glycine plausible, assuming it co-desorbs with water. After the first detection of nitrogen hydrides in Sgr B2 (Goicoechea et al. 2004), a complex region encompassing a variety of physical conditions and in particular shocks, the new detection with Herschel/HIFI of $\mathrm{NH}$ (and $\mathrm{ND}$ ), $\mathrm{NH}_{2}$, and $\mathrm{NH}_{3}$ towards the external envelope of the protostar IRAS 16293-2422 (Hily-Blant et al. 2010; Bacmann et al. 2010) has not improved our knowledge of the interstellar chemistry of nitrogen hydrides. In a recent publication, Le Gal et al. (2014) develop a new model that could explain the formation of these hydrides in the gas phase while some of the modelers mentioned above claim surface formation. Concerning oxygen, one of the conclusions of deep observations of $\mathrm{O}_{2}$ towards NGC 1333 IRAS 4A protostar (Yildiz et al. 2013) is that the observed low molecular oxygen abundance is due to the freeze-out of atomic $\mathrm{O}$ onto grains. All these observations lead to the conclusion that on the grains in dense clouds surrounding protostellar cores, there can be $\mathrm{NH}_{3}$ and $\mathrm{O}$ present.

On the experimental side, two important facts are of interest. First, concerning $\mathrm{NH}_{3}$, it has been experimentally demonstrated (Hidaka et al. 2011) that the successive hydrogenation of $\mathrm{N}$ atoms trapped in a solid $\mathrm{N}_{2}$ matrix at $10 \mathrm{~K}$ leads to $\mathrm{NH}_{3}$ formation. Second, the formation of $\mathrm{NH}_{2} \mathrm{OH}$ has been observed after irradiating an ammonia-water ice at $90-130 \mathrm{~K}$ with UV photons (Nishi et al. 1984), and at 10 and $50 \mathrm{~K}$ with $5 \mathrm{keV}$ electrons (Zheng \& Kaiser 2010). The chemical processing of $\mathrm{H}_{2} \mathrm{O}-\mathrm{NH}_{3}$ ices induced by heavy ions (Bordalo et al. 2013) leads also, by radiolysis, to the formation of $\mathrm{N}_{2} \mathrm{O}, \mathrm{NO}, \mathrm{NO}_{2}$, and $\mathrm{NH}_{2} \mathrm{OH}$. As mentioned above, (Blagojevic et al. 2003) showed that the reaction of hydroxylamine ions, $\mathrm{NH}_{2} \mathrm{OH}^{+}$, with acetic or propanoic acid makes ionized glycine and alanine. Much earlier, from about 1960 to mid 1980s, the $\mathrm{NH}_{3}+\mathrm{O}$ reaction has been the subject of numerous experiments reviewed by (Cohen 1987). They have been performed at moderate temperature $(450$ to $850 \mathrm{~K}$ ) in flowing and static systems (Perry 1984; Baulch et al. 1984) where ground state $\mathrm{O}\left({ }^{3} \mathrm{P}\right)$ is obtained by laser photolysis, or at higher temperatures $(850$ to $2200 \mathrm{~K})$ in flames (Fenimore \& Jones 1961) or in shock tube experiments (Fuiii et al. 1986). Even if such a reaction has never been studied at very low temperature, it has been proposed that excited $\mathrm{NH}_{3} \mathrm{O}$ intermediate could rearrange to give stable hydroxylamine $\mathrm{NH}_{2} \mathrm{OH}$ (Baulch et al. 1984). The formation of complex organic molecules involving atomic addition reactions on the cold surface of ices-covered interstellar grains in diverse environments, has been extensively reviewed recently (Herbst \& van Dishoeck 2009). As NO has been detected in the gas phase towards a few high-mass starforming regions, it has been suggested that hydroxylamine could be formed in the gas phase through nonenergetic hydrogenation of NO ice under dark cloud conditions (Charnley et al. 2001). But recently, an NO ice hydrogenation surface reaction at low temperature has shown that an efficient route to $\mathrm{NH}_{2} \mathrm{OH}$ formation was also possible with interstellar relevant ices (Congiu et al. 2012a,b; Fedoseev et al. 2012). It takes place at 10 to $15 \mathrm{~K}$ at both NO submonolayer and multilayer cover- age. Such a process occurs without external energetic input but implies several successive steps of hydrogenation. It is worth mentioning a crossed molecular beam study of the $\mathrm{O}\left({ }^{1} \mathrm{D}\right)+\mathrm{NH}_{3}$ reaction at $295 \mathrm{~K}$ (Shu et al. 2001) forming vibrationally excited $\mathrm{NH}_{2} \mathrm{OH}$ with a subsequent dissociation into two different reaction channels: $\mathrm{OH}+\mathrm{NH}_{2}$ and $\mathrm{NHOH} / \mathrm{NH}_{2} \mathrm{O}+\mathrm{H}$ with $90 \%$ and $10 \%$ yield respectively. This reaction has been also studied theoretically (Wang et al. 2004). Finally, very recently, hydroxylamine have been formed in a two-step mechanism through the reaction of ammonia with hydroxyl radicals in a Neon matrix at $4 \mathrm{~K}$ (Zins \& Krim 2014).

However, most of the objects mentioned above, if not all, either in high-mass or low-mass star-forming regions as well as in proto-stellar cores, share the common characteristic of having a large abundance of $\mathrm{NH}_{3}$ (and also $\mathrm{H}_{2} \mathrm{O}$ ), in any case far higher than the NO abundance. This latter molecule appears to be present in a restricted number of objects relating to high-mass star-forming regions. The abundance of $\mathrm{NH}_{3}$ is asserted in observations of low-mass young stellar objects (Bottinelli et al. 2010) and even dark clouds (as the Bok globule Barnard 68 (Di Francesco et al. 2002)) and comets (as very recently detected on $67 \mathrm{P} / \mathrm{C}-\mathrm{G}$ by the ROSINA instrument aboard ROSETTA (Altwegg 2014)). Then the formation process in star-forming regions of hydroxylamine we present in this paper is a very likely more direct (single step) and realistic (considering the species involved) mechanism, compared to the one already known (Congiu et al. $2012 \mathrm{~b}$ ).

Actually this process is well suited with respect to to hot-cores and protostellar cores. In addition to $\mathrm{N}$ resulting from $\mathrm{N}_{2}$ dissociation under cosmic-rays irradiation, atomic $\mathrm{O}$ is also present for the same reason. Both $\mathrm{N}$ and O stick on the colder bare or ices covered grains, the ones deep into the cloud surrounding the core $\left(\mathrm{A}_{v} \sim 15-20\right)$. A very simplistic scheme could then be the following: (i) Very cold grains are covered by atoms ( $\mathrm{H}, \mathrm{O}$, and $\mathrm{N})$, either on the surface or in bulk, (ii) when the temperature begins to increase $(>5-10 \mathrm{~K}), \mathrm{H}$ atoms become mobile and form $\mathrm{NH}_{3}$ (and $\mathrm{H}_{2} \mathrm{O}$ ) by hydrogenation surface reactions, (iii) as the temperature continues to increase ( $>14 \mathrm{~K}$ in interstellar space conditions - corresponding in laboratory experiments to $>20-25 \mathrm{~K})$ ), $\mathrm{O}$ atoms become mobile to finally react with $\mathrm{NH}_{3}$ (and $\mathrm{H}_{2} \mathrm{O}$ ) already formed to to produce in particular $\mathrm{NH}_{2} \mathrm{OH}$ through an insertion mechanism.

In this paper we present experimental evidence of the formation process of hydroxylamine via oxidation of $\mathrm{NH}_{3}$ on dust grain analogs (Section 3). The experimental findings are then used in a 3-phase gas-grain model of a dark cloud (Section 4). A comparison of $\mathrm{NH}_{2} \mathrm{OH}$ formation mechanisms and a discussion about suitable environments where $\mathrm{NH}_{2} \mathrm{OH}$ could be observed are presented in Sections 5 and 6.

\section{EXPERIMENTAL SETUP}

\subsection{Apparatus}

The apparatus was described elsewhere (He et al.2011; Jing et al. 2013); here we summarize the main features that are important for this study. The experiments were carried out in an ultra-high vacuum main chamber connected to an atomic/molecular beam line. The 
main chamber is pumped by a combination of a cryopump, turbomolecular pump, and ion pump and can reach $1.2 \times 10^{-10}$ torr after a bake-out. At the center of the chamber there is a $1 \mu \mathrm{m}$ thick amorphous silicate thin film sample grown on a $1 \mathrm{~cm}^{2}$ gold plated copper disk by electron beam physical vapor deposition. The detailed preparation and characterization of the sample can be found in Jing et al. (2013). The sample can be cooled to $8 \mathrm{~K}$ by a liquid helium-cooled sample holder and be heated up to $450 \mathrm{~K}$ by a cartridge heater behind the sample. At the beginning of each day, the sample is heated up to $400 \mathrm{~K}$ to clean it before cooling it down. The chamber pressure after cooling down is about $5 \times 10^{-11}$ torr. During exposure of the sample to the atomic/molecular beam, the pressure increases to about $1 \times 10^{-10}$ torr. At this pressure, the amount of water that stick on the sample from the chamber background gas is negligible. A triple-pass quadrupole mass spectrometer (QMS) is mounted on a rotary platform to record desorbed species from the sample surface or to measure the beam composition. The QMS is placed in a differentially pumped enclosure and is fitted with a cone aimed at a sample. The distance between the tip of the cone and the sample can be changed by repositioning the sample via a XYZ sample holder manipulator. This arrangement prevents products loosely adsorbed on sample holder parts from reaching the QMS, thus reducing the background level and improving the signal/noise. A radio-frequency dissociation source is mounted in the first stage of the triple differentially pumped beam line. A mass flow controller (Alicat MCS-5SCCM) is used to ensure stable gas flow.

\subsection{Beam flux calibration}

The beam fluxes are calibrated by TPD experiments. With the the amorphous silicate sample kept at $70 \mathrm{~K}$, ammonia gas is introduced from the molecular beam line. The gas flow is set to $0.3 \mathrm{sccm}$ on the mass flow controller. After ammonia exposure, the sample is cooled down to below $40 \mathrm{~K}$ and then heated up at $1 \mathrm{~K} / \mathrm{s}$ to about $200 \mathrm{~K}$ to do a TPD. In both ammonia exposure stage and TPD stage, the gas phase molecules are measured using the QMS. Since ammonia fragments in the QMS ionizer, both mass $17 \mathrm{amu}\left(\mathrm{NH}_{3}^{+}\right)$and $16 \mathrm{amu}$ $\left(\mathrm{NH}_{2}^{+}\right)$are measured. The fragmentation of ammonia into $\mathrm{NH}^{+} / \mathrm{N}^{+}$(mass 15/14 amu) is found to be negligible by direct beam measurements. A series of TPD experimental runs with different ammonia doses are performed. The TPD spectra are shown in Figure 1. We calibrate the $\mathrm{NH}_{3}$ coverage by analyzing the shape of the TPD profile. In the submonolayer region the TPD peak temperature should shift to lower values, because in the low coverage region molecules occupy the deep sites (higher desorption energies). Here a layer is actually an equivalent layer, since it is unclear whether ammonia would form clusters or islands on the surface; little experimental evidence support the existence of clusters or islands. As the deep sites get filled up with increasing exposure, the TPD profile and peak temperature shift to lower temperatures (He \& Vidali 2014). In the multilayer region, the TPD spectra should show a common leading edge (Kolasinki 2008), which is a typical first order desorption behavior. The trend of TPD traces shown in Figure 1 is in agreement with the one obtained by

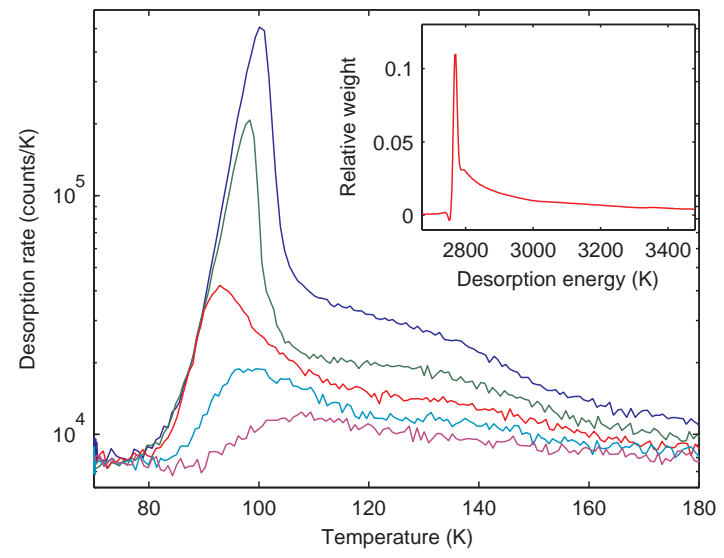

Figure 1. TPDs with different exposure of $\mathrm{NH}_{3}$ at $70 \mathrm{~K}$. The exposure times are, from bottom to top, $0.5,1,2,4$, and 8 minutes, respectively. Desorption energy distribution of $\mathrm{NH}_{3}$ calculated from the 2 minutes exposure curve (1 ML deposition) is in the inset.

Bolina et al. (2005) for $\mathrm{NH}_{3}$ desorption from a graphite surface. With the current experimental settings, $1 \mathrm{ML}$ $\mathrm{NH}_{3}$ coverage is achieved by 2 minutes exposure with $0.3 \mathrm{sccm}$ gas flow. Thus the $\mathrm{NH}_{3}$ flow is $0.5 \mathrm{ML} /$ minute, or equivalently, $5 \times 10^{14} \mathrm{~cm}^{-2}$ minute ${ }^{-1}$, assuming $1 \mathrm{ML}$ $\sim 10^{15} \mathrm{~cm}^{-2}$. Following the procedures as described in He \& Vidali (2014), the desorption energy distribution of $\mathrm{NH}_{3}$ from amorphous silicate surface can be obtained by direct inversion of the 1 ML trace in Figure 1 . The resulted distribution is shown in the inset of Figure 1 Additional TPD runs are performed at a surface temperature of $10 \mathrm{~K}, 30 \mathrm{~K}$, and $50 \mathrm{~K}$. They show almost identical TPD curves as the one at $70 \mathrm{~K}$. This suggests that the sticking of ammonia on the silicate surface at 70 $\mathrm{K}$ is unity, and the desorption rate at $70 \mathrm{~K}$ is negligible.

In the atomic oxygen exposure, the $\mathrm{O}_{2}$ gas flow passing through the flow controller is set to $0.1 \mathrm{sccm}$. At this flow the dissociation rate of $\mathrm{O}_{2}$ is measured to be $42 \%$. The calibration of oxygen flux needs to be done differently from ammonia because $\mathrm{O}_{2}$ is volatile and the sticking is not necessarily unity. The direct beam intensities of $\mathrm{O}_{2}$ gas at both $0.3 \mathrm{sccm}$ and $0.1 \mathrm{sccm}$ are measured. Since it is known that $0.3 \mathrm{sccm}$ corresponds to $0.5 \mathrm{ML} /$ minute, the flow at $0.1 \mathrm{sccm}$ can be calculated correspondingly. The $\mathrm{O}_{2}$ flow at $0.1 \mathrm{sccm}$ is about $0.34 \mathrm{ML} /$ minute $\left(3.4 \times 10^{14} \mathrm{~cm}^{-2}\right.$ minute $\left.{ }^{-1}\right)$. When the $\mathrm{RF}$ is on, the beam intensities of $\mathrm{O}$ and $\mathrm{O}_{2}$ are $0.29 \mathrm{ML} /$ minute $\left(2.9 \times 10^{14} \mathrm{~cm}^{-2}\right.$ minute $\left.{ }^{-1}\right)$ and $0.20 \mathrm{ML} /$ minute $\left(2.0 \times 10^{14} \mathrm{~cm}^{-2}\right.$ minute $\left.^{-1}\right)$, respectively. With the radio frequency $(\mathrm{RF})$ power on and the oxygen sent into the dissociation source, beam contamination is checked. The main contaminant is NO (mass $30 \mathrm{amu}$ ) due the small leak of air into the dissociation source. The NO (mass 30) signal is less than $3 \%$ of the O signal, which is trivial in the context of the experiments performed here.

\subsection{Experimental procedures}

The ammonia oxidation reactions were studied in ammonia and atomic oxygen sequential exposure TPD experiments. The surface was covered with ammonia before introducing atomic oxygen. Two ammonia coverages were used, $2 \mathrm{ML}$ and $1 / 4 \mathrm{ML}$, representative of multilayer 


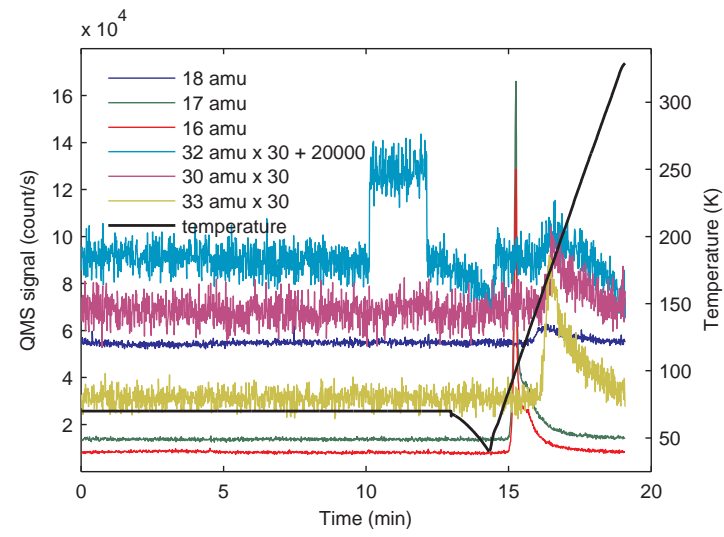

Figure 2. A typical TPD of a $\mathrm{NH}_{3}+\mathrm{O}$ sequential exposure experiment at $70 \mathrm{~K}$ in which the QMS records simultaneously multiple signals. The temperature ramp (black line) shows that after the exposure is terminated, the sample is cooled to $40 \mathrm{~K}$ before the heating is started.

coverages and submonolayer coverages. After preparation of the ammonia sample, the residual ammonia in the beam line and dissociation source was cleaned by flushing the beam line several times using oxygen. This ensures that almost no ammonia was mixed with oxygen. The sample temperature in the exposure stage was chosen to be $70 \mathrm{~K}$ so that $\mathrm{O} / \mathrm{O}_{2} / \mathrm{O}_{3}$ does not stick onto the surface while the sticking of $\mathrm{NH}_{3}$ is unity. After the exposure stage, the surface was cooled down to below 40 $\mathrm{K}$ and then heated up linearly at $1 \mathrm{~K} / \mathrm{s}$ to above $320 \mathrm{~K}$ to desorb species from the surface. The QMS recorded simultaneously the signals of various masses during both exposure and TPD stages. Figure2 2 shows the TPD spectra of a typical experimental run.

\section{RESULTS AND ANALYSIS}

\subsection{Multilayer $\mathrm{NH}_{3}+\mathrm{O}$}

In the $\mathrm{NH}_{3}+\mathrm{O}$ sequential exposure TPD experiments, mass $16,17,18,30,32$, and 33 amu were measured by the QMS, see Figure 2. Mass $16 \mathrm{amu}$ is due to the fragmentation of $\mathrm{NH}_{3}$ in the QMS ionizer; mass $17 \mathrm{amu}$ is due to $\mathrm{NH}_{3}$ and fragmentation of $\mathrm{H}_{2} \mathrm{O}$; mass $32 \mathrm{amu}$ is due to $\mathrm{O}_{2}$ and fragments of $\mathrm{O}_{3}$. Mass 30 amu could be due to NO from the beam line (but the amount is small), or fragments of $\mathrm{NH}_{2} \mathrm{OH}$; mass 33 amu could be due to $\mathrm{NH}_{2} \mathrm{OH} . \mathrm{HO}_{2}$ has the same mass but it is unlikely to be there because of the lack of detection of the products it fragments into. The mass 33 amu peak centered at around $180 \mathrm{~K}$ is accompanied by mass $30 \mathrm{amu}$ and very small signals with masses 16, 17 and 32 amu at the same temperature; this suggests that the mass $33 \mathrm{amu}$ peak at $180 \mathrm{~K}$ is due to $\mathrm{NH}_{2} \mathrm{OH}$. This agrees with the TPD peak attribution in Congiu et al. (2012a) (in the range of 160-200 K, peaking at $\mathrm{T} \sim 190 \mathrm{~K}$ ) obtained in an NO hydrogenation experiment and confirmed by infrared measurements (RAIRS). Mass $18 \mathrm{amu}$ is due to water.

Figure 3 shows the mass $17 \mathrm{amu}\left(\mathrm{NH}_{3}\right)$ signal after depositing various doses of $\mathrm{O}$ on top of 2 ML (4 minutes exposure) $\mathrm{NH}_{3}$. As the $\mathrm{O}$ exposure time increases, the $\mathrm{NH}_{3}$ peak decreases and the peak position also shifts to higher temperatures. This is because the top layer of $\mathrm{NH}_{3}$ is gradually converted to $\mathrm{NH}_{2} \mathrm{OH}$ or other products, thus hindering the desorption of $\mathrm{NH}_{3}$ molecules under-



Figure 3. Mass $17 \mathrm{amu}$ QMS signal of the TPD from an ice consisting of $\mathrm{NH}_{3}(4 \mathrm{~min}$ or $2 \mathrm{ML}$ ) followed by exposure to various doses of $\mathrm{O}$ at $70 \mathrm{~K}$.

neath. After about 6 minutes of $\mathrm{O}$ exposure, the first peak almost disappears, indicating the top layer of $\mathrm{NH}_{3}$ is almost all converted to $\mathrm{NH}_{2} \mathrm{OH}$ or other products.

In Figure 4 two desorption peaks, peak $\mathrm{A}$ and peak B, are visible. Peak A shows up at $\mathrm{O}$ exposure as low as 0.5 minutes. This indicates that the ammonia oxidation reaction is efficient. As the $\mathrm{O}$ exposure increases from 0 to 4 minutes, the area of peak $\mathrm{A}$ increases at first, but then it decreases from 4 minutes to 16 minutes of $\mathrm{O}$ exposure. Peak $\mathrm{A}$ is followed, at larger $\mathrm{O}$ exposures, by peak B at $\sim 260 \mathrm{~K}$. Notice that peak B starts to appear at 4 minutes of $\mathrm{O}$ exposure time at the expense of peak A. As mentioned above, peak A falls in the temperature range of the TPD mass 33 amu peak obtained by Congiu et al. (2012a). Therefore we attribute peak A to the desorption of $\mathrm{NH}_{2} \mathrm{OH}$. Concerning peak B, however, in a subsequent paper on an $\mathrm{NO}_{2}$ hydrogenation experiment (Ioppolo et al. 2014), a mass 33 peak, confirmed by infrared measurement spectra, appears at a temperature $\mathrm{T} \sim 245 \mathrm{~K}$, close to the temperature of our second peak (peak B in our Figure 4). Nevertheless, oddly there is no mention in their paper of a peak A at $160 \mathrm{~K}$.

To find out what peak B represents, we show in Figure 5 a comparison of TPD traces for various masses for 2 ML of $\mathrm{NH}_{3}+8$ or 16 minutes of $\mathrm{O}$ exposure, see left and right panels of Figure 5 respectively. Peak A and peak $\mathrm{B}$ are marked by vertical lines. In both panels of Figure 5. peak A (see trace of mass $33 \mathrm{amu}$ ) is accompanied by desorption of mass $30 \mathrm{amu}, 17 \mathrm{amu}$, and $16 \mathrm{amu}$, while this is not true for peak B. This suggests that peak B is not due to $\mathrm{NH}_{2} \mathrm{OH}$. It could be the product of a fragmentation of a dimer (Del Bene 1972) or of an oxidation product of $\mathrm{NH}_{2} \mathrm{OH}$. At about $280 \mathrm{~K}$, there is a peak C showing up for mass $30 \mathrm{amu}, 17 \mathrm{amu}$, and $16 \mathrm{amu}$, but only when the $\mathrm{O}$ dose is high (16 minutes of $\mathrm{O}$ exposure), see right panel of Figure 5. It could be due to a yet unidentified product formed in a further oxidation of $\mathrm{NH}_{2} \mathrm{OH}$. Peak $\mathrm{B}$ and peak $\mathrm{C}$ differs in both position and shape, therefore they should be attributed to different species.

\subsection{Submonolayer $\mathrm{NH}_{3}+\mathrm{O}$}

Experiments were also carried out at submonolayer $\mathrm{NH}_{3}$ coverage. We exposed the silicate sample to $\mathrm{NH}_{3}$ 


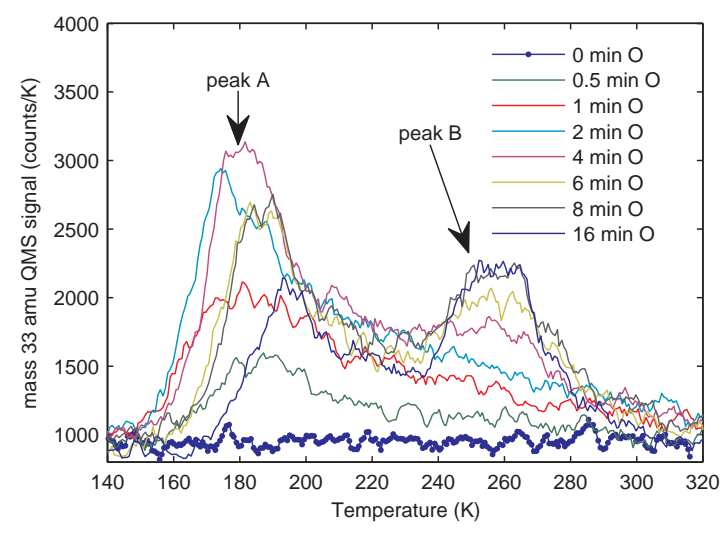

Figure 4. As in Figure 3 but for QMS mass $33 \mathrm{amu}$ signal.

for 0.5 minutes, or about $1 / 4$ of a layer, with the same procedures as the ones followed to obtain the data in Figure 1. This was followed by exposure to O. Mass $16 \mathrm{amu}$ was chosen to represent the $\mathrm{NH}_{3}$ amount because the mass 17 amu signal has a non-trivial contribution from water fragmentation when the $\mathrm{NH}_{3}$ signal is weak. In Figure 6 we show the integrated mass 16 amu TPD trace for different exposures to $\mathrm{O}$. With $\mathrm{O}$ exposure from 0 to 2 minutes, the amount of $\mathrm{NH}_{3}$ follows more or less an exponential decay. This is because when the $\mathrm{O}$ amount is small, oxidation dominates and the $\mathrm{NH}_{3}$ destruction rate is proportional to the $\mathrm{NH}_{3}$ amount. With a further increase in $\mathrm{O}$ exposure, the $\mathrm{NH}_{3}$ decay does not follow a simple exponential decay anymore because of possible secondary reactions. In Figure [6 a straight line is fitted to the $\log _{e}$ plot from 0 to 2 minutes. The slope is $-0.46 \pm 0.06$ minute $^{-1}$. In an exponential decay, the amount of $\mathrm{NH}_{3}$ on the surface should follow $\exp (-\sigma \phi t)$, where $\sigma$ and $\phi$ are the reaction cross-section area and the beam flux, respectively. From it, we obtain the cross section $\sigma=-$ slope $/ \phi=(1.6 \pm 0.2) \times 10^{-15} \mathrm{~cm}^{2}$.

\subsection{Reaction energy barrier of $\mathrm{NH}_{3}+\mathrm{O}$}

In typical ISM conditions, it is unlikely that situations arise similar to the ones that led to the appearance of the second TPD peak of mass 33 amu at high temperature attributed to a subsequent oxidation process. Therefore we focus on the first oxidation:

$$
\mathrm{NH}_{3}+\mathrm{O} \rightarrow \mathrm{NH}_{2} \mathrm{OH}
$$

When the surface is fully covered with $\mathrm{NH}_{3}$ and the surface temperature is $70 \mathrm{~K}, \mathrm{O}$ could react with $\mathrm{NH}_{3}$ also via the Eley-Rideal or hot-atom mechanisms, i.e. reaction without complete thermal accommodation of the oxygen atom. Based on prior experiments (He et al. 2014), we assume that at $70 \mathrm{~K}$ the residence time of $\mathrm{O}$ on $\mathrm{NH}_{3}$ ice is negligible. We also assume that all the reactions take place during the exposure stage instead of the TPD stage, because at $70 \mathrm{~K}$ the mobility of oxygen should be relatively high. The degree of conversion of $\mathrm{NH}_{3}$ can be gauged by looking at what remains of the $\mathrm{NH}_{3}$ layers after various doses of oxygen. Figure 3 shows the TPD mass 17 signal $\left(\mathrm{NH}_{3}\right)$ for various doses of $\mathrm{O}$. The $\mathrm{NH}_{3}$ dose is fixed at 4 minutes, which is equivalent to about $2 M L$. As the $\mathrm{O}$ exposure time increases, the $\mathrm{NH}_{3}$ peak decreases, and the peak position also shifts to higher temperatures. This is because the top layer of
$\mathrm{NH}_{3}$ is gradually converted to $\mathrm{NH}_{2} \mathrm{OH}$ or other products, thus preventing $\mathrm{NH}_{3}$ molecules underneath from desorbing. After about 6 minutes of $\mathrm{O}$ exposure, the first peak almost disappears, indicating that the top layer of $\mathrm{NH}_{3}$ is almost all converted to $\mathrm{NH}_{2} \mathrm{OH}$ or other products. Thus it takes at most $6 \mathrm{~min}$ of $\mathrm{O}(1.74 \mathrm{ML})$ to convert $1 \mathrm{ML}$ of $\mathrm{NH}_{3}$, suggestion an efficiency for the reaction of each oxygen atom of at least $0.575(=1 / 1.74)$.

Using this efficiency, an upper limit for the activation energy barrier, $E_{\mathrm{A}}$, for the $\mathrm{O}+\mathrm{NH}_{3}$ reaction may be estimated.

We firstly assume that the incoming oxygen atom is thermalized on the surface, before overcoming the activation energy barrier to reaction. This thermalization is the most likely immediate outcome of the impact, except in a small minority of "direct-hit" trajectories, as the oxygen atom dissipates energy as it is drawn into the multi-dimensional potential of the surface. The atom is nevertheless available for immediate reaction if the activation energy barrier can then be overcome; in such a case, the reaction may be classed as Eley-Rideal, as it is not mediated by a diffusion process.

Assuming that the main loss mechanism for an oxygen atom on a pure $\mathrm{NH}_{3}$ surface is either reaction with $\mathrm{NH}_{3}$ or thermal desorption, the reaction efficiency may be formulated thus:

$1 / 1.74=\exp \left[-E_{\mathrm{A}} / T\right] /\left(\exp \left[-E_{\mathrm{A}} / T\right]+\exp \left[-E_{\mathrm{des}}(\mathrm{O}) / T\right]\right)$

where $E_{\text {des }}(O)$ is the desorption energy for an oxygen atom and $\mathrm{T}$ is the surface temperature. Adopting $E_{\text {des }}(\mathrm{O})=1500 \mathrm{~K}$ and $\mathrm{T}=70 \mathrm{~K}$, this expression produces a value $E_{A}\left(\mathrm{O}+\mathrm{NH}_{3}\right)=1479 \mathrm{~K}$.

This value constitutes an upper boundary of the reaction energy barrier because we assume a sticking coefficient of unity, and thence that each oxygen atom that reaches the surface participates in a reaction. If this is not the case, then the reaction energy barrier for the first oxidation should be even smaller.

The above treatment does not consider the possibility that surface oxygen atoms could meet each other and react, reducing the efficiency of the reaction. Under conditions in which such reactions could be important, then in order for the $\mathrm{O}+\mathrm{NH}_{3}$ reaction to be efficient and the $\mathrm{O}+\mathrm{O}$ reaction to be minimized, the reaction barrier for $\mathrm{O}+\mathrm{NH}_{3}$ would need to be lower than the oxygen diffusion barrier. This quantity has not been measured in the laboratory, but there are rulesof-thumb based on experimental data from other systems. The ratio of the diffusion energy barrier to the desorption energy is typically found to be $\sim 0.3$ for crystalline surfaces (Bruch et al. 2007). For rough surfaces, values between 0.5 and 0.8 have been used (Katz et al. 1999; Garrod \& Herbst 2006), although Garrod \& Pauly (2011) found that values lower than $\sim 0.4$ were most consistent with observed interstellar extinction thresholds for $\mathrm{H}_{2} \mathrm{O}, \mathrm{CO}$ and $\mathrm{CO}_{2}$ ices. Garrod (2013) adopted a value of 0.35 , which would yield an upper limit on the $\mathrm{O}$ $+\mathrm{NH}_{3}$ activation energy barrier as low as $525 \mathrm{~K}$.

\subsection{Control experiments}

Control experiments were carried out to verify whether ammonia react with molecular oxygen or ozone. We ex- 

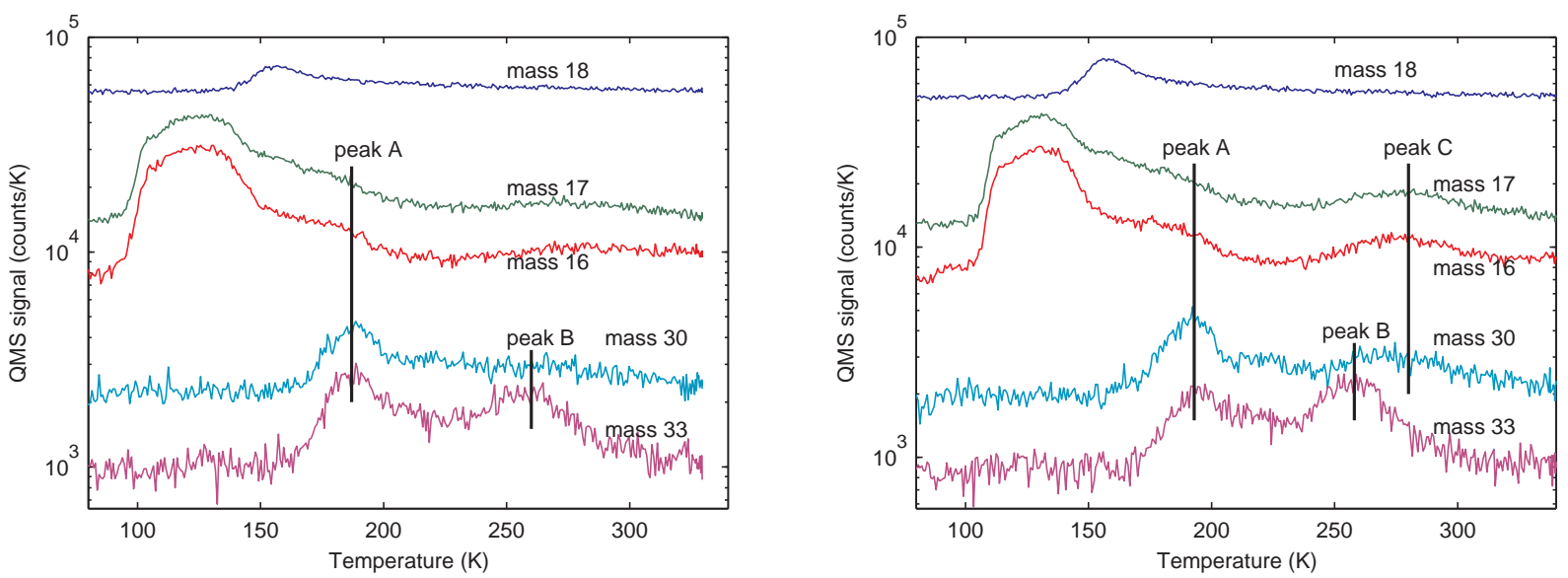

Figure 5. QMS signal of the TPD from an ice of $\mathrm{NH}_{3}(4 \mathrm{~min})+8$ (left) and 16 (right) minutes of $\mathrm{O}$ exposure.

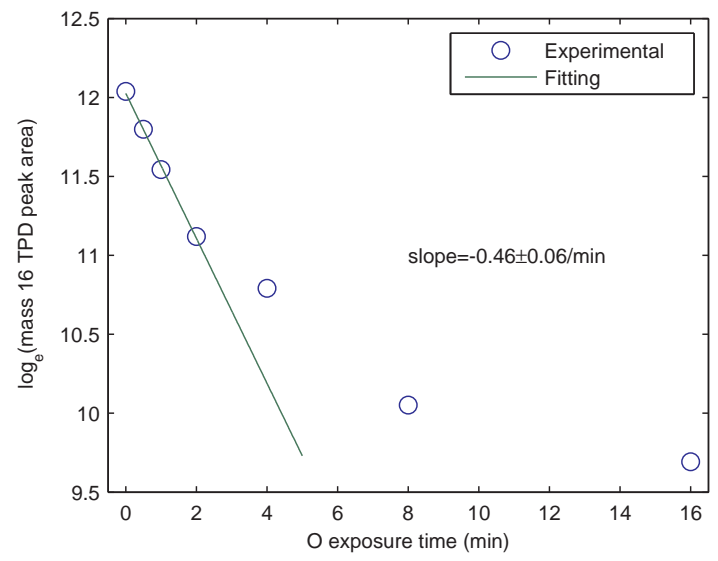

Figure 6. Integrated TPD signs mass $16 \mathrm{amu}$ from a deposit of $\mathrm{NH}_{3}(0.5$ minutes or $1 / 4$ of a ML) + various exposures of $\mathrm{O}$ at 70 $\mathrm{K}$.

posed the ammonia layers to $\mathrm{O}_{2}$ and found no mass 33 or 30 amu peaks in the TPD, signifying that there has been no conversion of ammonia to hydroxylamine in the presence of molecular oxygen. We also checked the reactivity of ozone with ammonia. Ozone was prepared on a clean silicate following the procedure described in He et al. (2014); then, 2 ML of ammonia were deposited on it. Again, there was no mass 33 or 30 amu peaks in the following TPD, see Figure 7

\section{MODELING}

In order to test the astrophysical importance of the $\mathrm{O}+\mathrm{NH}_{3} \rightarrow \mathrm{NH}_{2} \mathrm{OH}$ reaction investigated here, we incorporate this surface mechanism into the recent threephase gas-grain chemical kinetics model MAGICKAL (Garrod 2013). The model parameters used here are intended to approximate those present under dark cloud conditions, during which significant ice mantles are formed on the surfaces of dust grains. The model utilizes the chemical network and initial elemental abundances used by Garrod (2013), assuming a generic, static dark-cloud gas density of $n_{\mathrm{H}}=2 \times 10^{4} \mathrm{~cm}^{-3}$ and visual extinction $A_{\mathrm{V}}=10$. A gas temperature of $10 \mathrm{~K}$ is used in all model runs. We assume a binding energy for atomic oxygen of $1500 \mathrm{~K}$, based on an experiment that measured the desorption energy of $\mathrm{O}$ from compact amorphous water ice (He \& Vidali 2014). Diffusion barriers are set to a uniform fraction of binding energies for

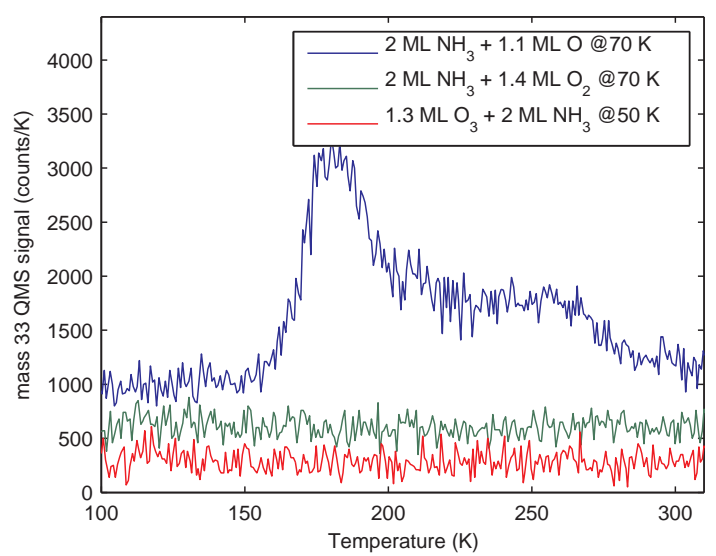

Figure 7. Mass 33 QMS signal in the TPD from $\mathrm{NH}_{3}+\mathrm{O}_{2}$ and $\mathrm{O}_{3}+\mathrm{NH}_{3}$ deposited at $70 \mathrm{~K}$ compared with that of $\mathrm{NH}_{3}+\mathrm{O}$ at $50 \mathrm{~K}$. Curves are offset for clarity.

all species, $E_{\text {diff }}=0.35 E_{\text {des }}$, following (Garrod 2013). Due to the low temperatures at which grain-surface ice mantles are formed, only surface chemistry is switched on in this model, although the three-phase treatment considers separate grain/ice surface and bulk mantle populations for all species (as well as gas-phase abundances). The three-phase model allows the composition of each layer within the ice mantle to be traced as it is deposited during the chemical evolution of the cloud (see Garrod \& Pauly (2011)). Only Langmuir-Hinshelwood (i.e. diffusional) processes are considered in this model, as the Eley-Rideal mechanism tends to require surface coverages of close to unity (in models of astrophysical grains) to compete with $\mathrm{L}-\mathrm{H}$ mediated reactions.

Reactions are also included in the network allowing $\mathrm{NO}$ molecules to be hydrogenated to $\mathrm{NH}_{2} \mathrm{OH}$ by the sequential addition of atomic H. Following (Garrod 2013), this includes a barrier-mediated reaction, $\mathrm{H}+\mathrm{HNO} \rightarrow$ $\mathrm{HNOH}$, as well as an alternative $\mathrm{H}$-abstraction branch, $\mathrm{H}+\mathrm{HNO} \rightarrow \mathrm{H}_{2}+\mathrm{NO}$, for which the barrier is assumed to be $1500 \mathrm{~K}$ in both cases. Following (Hasegawa et al. 1992), tunneling through activation energy barriers is treated using a rectangular barrier treatment, with a default width of $1 \AA$. The faster of tunneling and thermal penetration is used, according to the grain temperature.

The model described above is used to produce a grid of models for a range of dust-grain temperatures from 10 
- $20 \mathrm{~K}$, and using activation energy barriers, $E_{\mathrm{A}}$, for the $\mathrm{O}+\mathrm{NH}_{3} \rightarrow \mathrm{NH}_{2} \mathrm{OH}$ reaction ranging from $0-2500 \mathrm{~K}$. A smaller set of models is also run to compare the effects of $\mathrm{NH}_{2} \mathrm{OH}$ produced by this mechanism, with cases in which the barrier to the $\mathrm{H}+\mathrm{HNO} \rightarrow \mathrm{HNOH}$ reaction is altered.

\subsection{Model results}

Figure 8 panel (a), shows the composition of each layer in the grain mantle ice for the model run corresponding to $E_{\mathrm{A}}=0$ and $\mathrm{T}_{\text {grain }}=10 \mathrm{~K}$. Typically observed components are shown, along with $\mathrm{NH}_{2} \mathrm{OH}$. In this case, $\mathrm{NH}_{2} \mathrm{OH}$ production is relatively small, and all such production is a result of the hydrogenation of NO. At 10 $\mathrm{K}$, oxygen atoms are insufficiently mobile to be able to reach and react with $\mathrm{NH}_{3}$ before they are hydrogenated to produce water, even under the assumption of a zero barrier for the $\mathrm{O}+\mathrm{NH}_{3}$ reaction. Here, the majority of $\mathrm{NH}_{2} \mathrm{OH}$ is produced via $\mathrm{NO}$ hydrogenation, which is strongly peaked at late time/outer layers.

Panels (b) and (c) show the results at $14 \mathrm{~K}$, for activation energy barriers of $E_{\mathrm{A}}=1000$ and $2000 \mathrm{~K}$, respectively. At this temperature, oxygen atoms are sufficiently mobile to meet ammonia molecules on the surface before being hydrogenated. In the $E_{\mathrm{A}}=1000 \mathrm{~K}$ case, the reaction barrier is low enough (in comparison to the diffusion barrier for the oxygen atom to move away from the ammonia molecule) to allow reaction to occur with high efficiency. In this case, the majority of $\mathrm{NH}_{2} \mathrm{OH}$ is formed through this reaction, and $\mathrm{NH}_{2} \mathrm{OH}$ abundance closely follows that of $\mathrm{NH}_{3}$, never falling below a factor of ten lower than $\mathrm{NH}_{3}$ at any layer in the ice. $\mathrm{NH}_{2} \mathrm{OH}$ maintains a fairly steady abundance throughout the ice, up to around $1 \mathrm{Myr}(170 \mathrm{ML})$, but the $\mathrm{NH}_{2} \mathrm{OH}$ production is enhanced at all times/depths. In the $E_{\mathrm{A}}=2000 \mathrm{~K}$ case, the reaction barrier is too high to allow reaction before hydrogenation of oxygen occurs, making the $\mathrm{NH}_{3}+$ $\mathrm{O}$ reaction inefficient; here $\mathrm{NH}_{2} \mathrm{OH}$ production is again the result of NO hydrogenation.

Figure 9 shows the final (maximum) grain-mantle abundances of $\mathrm{NH}_{2} \mathrm{OH}$ as a function of total hydrogen in the cloud, for model runs assuming a range of grain temperatures from $10-20 \mathrm{~K}$, and activation energies for the $\mathrm{O}+\mathrm{NH}_{3}$ reaction ranging from $0-1500 \mathrm{~K}$. Results for the $\mathrm{E}_{\mathrm{A}}=500 \mathrm{~K}$ case are not shown, as they are identical to the $E_{\mathrm{A}}=0$ case; both values are sufficiently below the atomic oxygen diffusion barrier assumed in the model $(525 \mathrm{~K})$, such that the reaction with $\mathrm{NH}_{3}$ occurs at an efficiency close to unity, if the temperature is high enough to allow the oxygen atom to diffuse and reach an $\mathrm{NH}_{3}$ before it is hydrogenated and becomes immobile. This is the case for models of temperature $14 \mathrm{~K}$ and above; all models at $12 \mathrm{~K}$ or less produce the same results, with $\mathrm{NH}_{2} \mathrm{OH}$ produced by $\mathrm{NO}$ hydrogenation.

Assuming a barrier of $1000 \mathrm{~K}$, the reaction is still relatively efficient, falling by a factor $\sim 2$ at grain temperatures of $\sim 16 \mathrm{~K}$ and above, compared to the $E_{\mathrm{A}}=0 / 500$ $\mathrm{K}$ results. However, with a $1500 \mathrm{~K}$ barrier, reaction is inefficient, and yet higher barriers produce the same results; in such cases, $\mathrm{NH}_{2} \mathrm{OH}$ is produced overwhelmingly by $\mathrm{NO}$ hydrogenation. A run with the $\mathrm{O}+\mathrm{NH}_{3}$ barrier set to $1250 \mathrm{~K}$ allows this reaction to become slightly dominant over the NO hydrogenation mechanism, producing a small, though significant, increase in $\mathrm{NH}_{2} \mathrm{OH}$ production.

Models were also run to compare the case where the hydrogenation of $\mathrm{NO}$ by $\mathrm{H}$ atoms is assumed to occur either (i) without barriers or (ii) not to occur at all. Case (i), removal of the barrier to $\mathrm{H}+\mathrm{HNO} \rightarrow \mathrm{HNOH}$ (while the alternative hydrogen-abstraction branch retains its barrier of $1500 \mathrm{~K}$ ), in fact results in no appreciable difference from the runs that assume a barrier of $1500 \mathrm{~K}$ for $\mathrm{H}+\mathrm{HNO}$ (the zero-barrier results are thus not plotted explicitly in the figure). $\mathrm{NH}_{2} \mathrm{OH}$ production through this mechanism is therefore already at its highest efficiency.

The alternative regime, case (ii), in which the $\mathrm{H}+$ $\mathrm{HNO} \rightarrow \mathrm{HNOH}$ barrier is increased to arbitrary height, such that the reaction does not occur, results in negligible $\mathrm{NH}_{2} \mathrm{OH}$ production at $12 \mathrm{~K}$ and below, because all $\mathrm{NH}_{2} \mathrm{OH}$ formation is dependent on the $\mathrm{O}+\mathrm{NH}_{3}$ reaction, which is inefficient at these temperatures. Above 12 $\mathrm{K}, \mathrm{NH}_{2} \mathrm{OH}$ is formed efficiently via $\mathrm{O}+\mathrm{NH}_{3}$, and there is only a small difference between these results and those in which the $\mathrm{H}+\mathrm{HNO}$ reaction is allowed to occur at maximum efficiency.

\section{DISCUSSION}

Experiments of oxygen exposure on submonolayer and multilayer ammonia ices show an efficient formation of $\mathrm{NH}_{2} \mathrm{OH}$ with a reaction energy barrier that can be as low as $\sim 525 \mathrm{~K}$ and no higher than $1479 \mathrm{~K}$. The experiments were done on samples at $70 \mathrm{~K}$ to avoid contamination of molecular oxygen and ozone. At this temperature $\mathrm{NH}_{3}$ is stuck on the surface while $\mathrm{O}$ has a short residence time. Experiments have been performed at both bilayer and submonolayer coverage. Simulations were done to find what rates of hydroxylamine formation one would obtain in simulated dense cloud conditions. According to the chemical models, at dust temperatures around 14 $\mathrm{K}$ or higher, the $\mathrm{O}+\mathrm{NH}_{3}$ reaction is found to be an efficient mechanism for the production of $\mathrm{NH}_{2} \mathrm{OH}$. While temperatures this high are not the typically considered dark-cloud dust temperatures, it is certain that the dust in dark clouds must pass through such temperatures on their way to the $8-10 \mathrm{~K}$ that is more typically assumed. Furthermore, interstellar dust will again pass through the $\sim 14 \mathrm{~K}$ threshold at which this reaction becomes efficient as part of the evolution of hot cores.

\section{ASTROPHYSICAL IMPLICATIONS}

$\mathrm{NH}_{3}$ and $\mathrm{H}_{2} \mathrm{O}$ have been detected in the gas phase around transient protostellar cores or inferred for $\mathrm{O}$ from the strong $\mathrm{O}_{2}$ depletion. These cores are also classified as hot cores or hot corinos in the case of massive and medium/low mass nascent stars, respectively. These cores whose temperature can be as high as 100 $\mathrm{K}$ close to the protostellar source (and even $250 \mathrm{~K}$ for the densest ones) are much colder far from the core due to the strong extinction provided by the dusty molecular clumps in which they are embedded. A possible scenario for the coldest regions where $\mathrm{NH}_{3}$ and $\mathrm{O}$ are stuck on the grains and approaching the core is that in a first stage (at temperature in the $14-50 \mathrm{~K}$ range) $\mathrm{O}$ is released in the gas phase and is then able to collide with $\mathrm{NH}_{3}$ covered grains, inducing chemical reactions that can synthesize new species. These would later desorb from the grains when the core temperature increases. Alternatively, $\mathrm{NH}_{2} \mathrm{OH}$ may be produced at lower temperatures, 


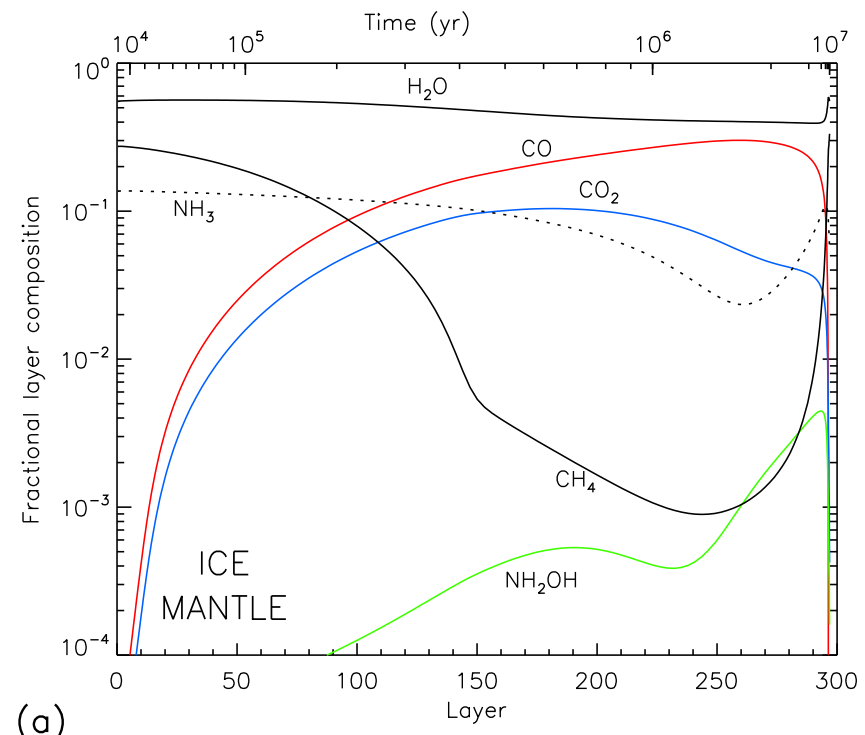

(a)

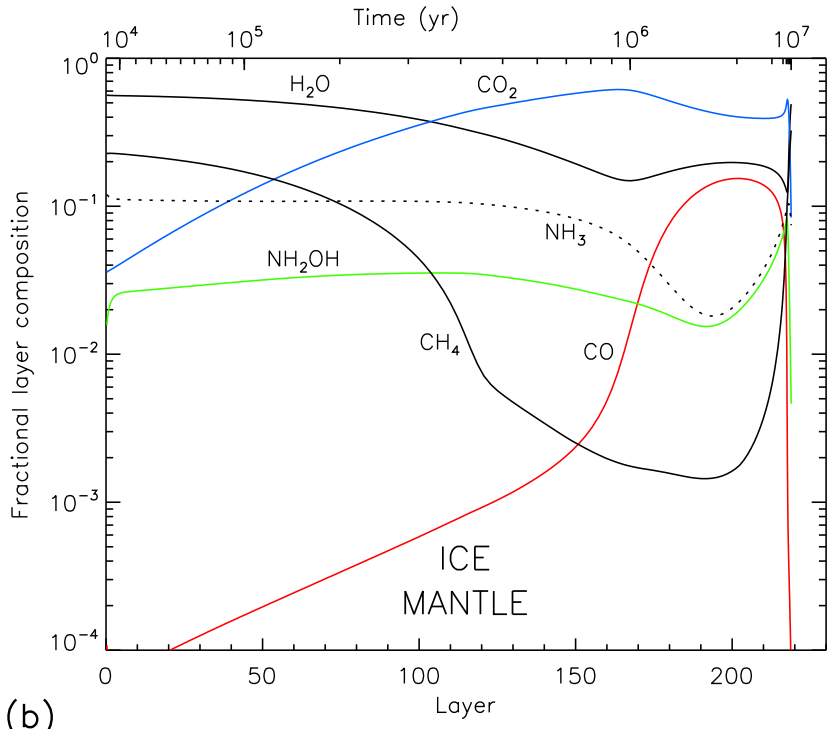

(b)

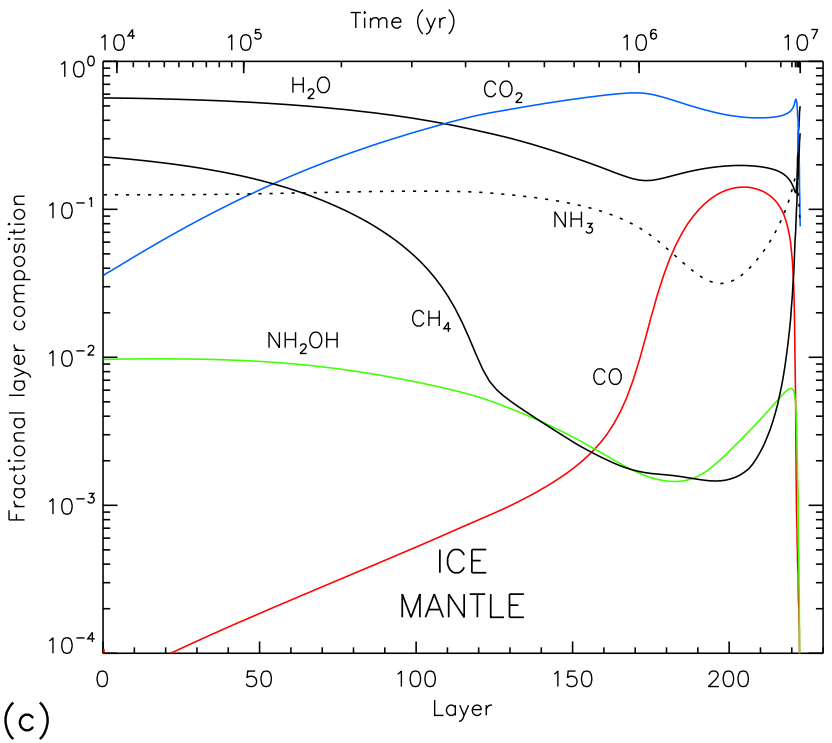

Figure 8. Fractional composition of ices within each layer as a function of depth into the ice, lower horizontal scale, or, equivalently, as a function of age of the cloud, top horizontal scale. Panel (a) The activation energy barrier for the $\mathrm{O}+\mathrm{NH}_{3}$ reaction is $0 \mathrm{~K}$ and the temperature of the ice is $10 \mathrm{~K}$. Panel (b) $E_{\mathrm{A}}=1000 \mathrm{~K}$ and $\mathrm{T}=14 \mathrm{~K}$. Panel (c) $E_{\mathrm{A}}=2000 \mathrm{~K}$ and $\mathrm{T}=14 \mathrm{~K}$.

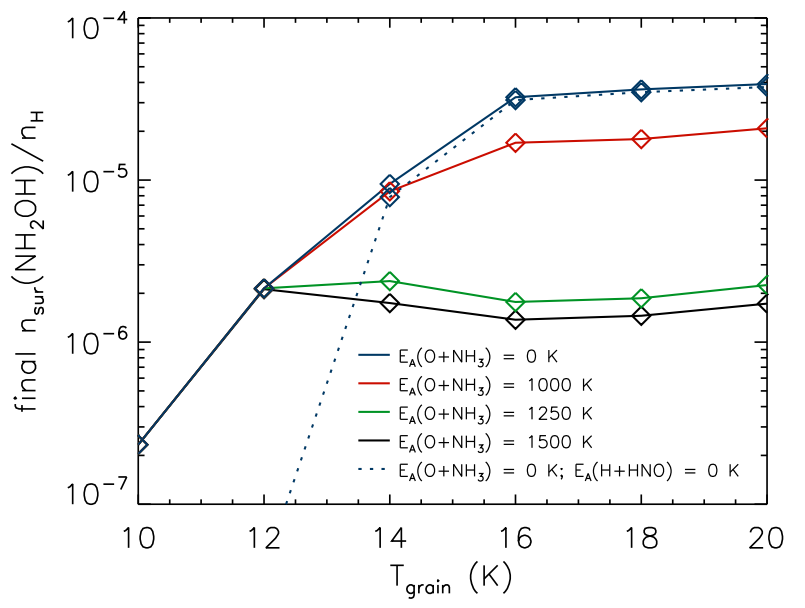

Figure 9. Final model abundances of ice-mantle $\mathrm{NH}_{2} \mathrm{OH}$ with respect to total hydrogen. The grain temperature is varied for a selection of activation-energy values for the $\mathrm{NH}_{3}+\mathrm{O}$ reaction. The dashed line shows the results assuming no barrier for either the $\mathrm{NH}_{3}+\rightarrow \mathrm{NH}_{2} \mathrm{OH}$ or $\mathrm{H}+\mathrm{HNOH} \rightarrow \mathrm{HNOH}$ reactions.

during the formation of the ice mantles, to be released at later times. (Models of such scenarios are beyond the scope of this primarily experimental study, but will be considered in future work).

Consequently, the present laboratory astrophysics experiment on $\mathrm{O}$ interaction with $\mathrm{NH}_{3}$ should be useful to interpret the observational data. Then the question is of the non-observation of hydroxylamine while, at the same time, it has been demonstrated that it can be made in the laboratory in conditions almost similar to those in protostellar cores. The upper limit relative abundance of $\mathrm{NH}_{2} \mathrm{OH}$ with respect to $\mathrm{H}_{2}$, as deduced from observations (Pulliam et al. 2012) is found to be in the $3 \times 10^{-9}$ to $8 \times 10^{-12}$ range, depending on the object. Interestingly, combining this result with the relative abundance of $\mathrm{NH}_{3}$ with respect to $\mathrm{H}_{2}$ in a high-mass star-forming region, $4.6 \times 10^{-8}$ (Battersby et al. 2014) (a value of 2 $\times 10^{-8}$ is already reported in the case of a cold dense cloud like TMC-1 (Turner 2000)), we deduce an average fractional abundance of $\mathrm{NH}_{2} \mathrm{OH}$ with respect to $\mathrm{NH}_{3}$ in the $0.7 \times 10^{-1}$ to $2 \times 10^{-4}$ range, close to the values deduced from the proposed model. Several reasons may explain the non-observation: the yield of such reactions could be quite low due to the narrow temperature range in which they have to take place as well as a possible spatial confinement of dusty clouds where the required temperature conditions for formation are fulfilled. In addition, recent observations (Sakai et al. 2013, 2014a, b; Sanna et al. 2014) have shown that the orientation of the protostellar core in the plane of the sky could be also important, particularly if it is associated to infall-disk-outflow systems and rotation. It could be expected that the amount of chemical species resulting from the $\mathrm{NH}_{3}+\mathrm{O}$ interaction will be almost equal both in high-mass and medium- to low-mass stars because of a higher species density in the first case and a longer interaction time in the other. All these conditions could explain the difficulty to observe such species as hydroxylamine that have, however, been made in the laboratory. ALMA could provide the required sensitivity and 
the sub-arcsecond angular resolution to observe them.

A recent paper (Cleeves et al. 2014) demonstrates, based on a physical and chemical model of the protoplanetary disk of the solar system, that the relative deuterated water abundance observed on Earth cannot be explained by its formation in the disk itself but should results in part from a primeval enrichment by interstellar ices. All species embedded in these ices may then have survived and have been injected into the disk and later on in the planetary system. This discovery confirms the paramount importance of observing prebiotic species like glycine or even smaller building blocks of such molecules like hydroxylamine, which would emphasize the possible extraterrestrial origin of life.

\section{ACKNOWLEDGMENTS}

This work is supported by the NSF Astronomy and Astrophysics Division (Grant No.1311958 to GV). We thank Dr. J. Brucato of the Astrophysical Observatory of Arcetri (Italy) for providing the sample used in these experiments and Zhirou Zhang, Jianming Shi, and Tyler Hopkins for technical help. RTG acknowledges the support of the NASA Astrophysics Theory Program (Grant No. NNX11AC38G).

\section{REFERENCES}

Altwegg, K. 2014, in ESA blog, http://blogs.esa.int/rosetta/2014/10/23/the-perfume-of-67pcg/

Bacmann, A., Caux, E., Hily-Blant, P., et al. 2010, A\&A, 521, L42 Barrientos, C., Redondo, P., Largo, L., Rayón, V. M., \& Largo, A. 2012, Astrophys. J., 748, 99

Battersby, C., Bally, J., Dunham, B., et al. 2014, ApJ, 786, 116

Baulch, D. L., Campbell, I. M., \& Hainsworth, R. 1984, J. Chem. Soc., Faraday Trans. 1, 80, 2525

Belloche, A., Menten, K. M., Comito, C., et al. 2008, A\&A, 482, 179

Blagojevic, V., Petrie, S., \& Bohme, D. K. 2003, Mon. Not. R. Astron. Soc., 339, L7

Bolina, A. S., Wolff, A. J., \& Brown, W. A. 2005, J. Chem. Phys., 122

Bordalo, V., da Silveira, E. F., Lv, X. Y., et al. 2013, Astrophys. J., 774, 105

Bottinelli, S., Boogert, A. C. A., Bouwman, J., et al. 2010, ApJ, 718,1100

Bruch, L., Diehl, R., \& Venables, J. 2007, Rev. Mod. Phys., 79, 1381

Caselli, P., Keto, E., Bergin, E. A., et al. 2012, Astrophys. J., 759, L37

Charness, C. 2013, Am. Astron. Soc. Meet. Abstr., 221

Charnley, S. B., Rodgers, S. D., \& Ehrenfreund, P. 2001, A\&A, 378,1024

Cleeves, L. I., Bergin, E. A., Alexander, C. M. O., et al. 2014, Science, 345,1590

Cohen, N. 1987, Int. J. Chem. Kinet., 19, 319

Congiu, E., Chaabouni, H., Laffon, C., et al. 2012b, J. Chem. Phys., 137, 054713

Congiu, E., Fedoseev, G., Ioppolo, S., et al. 2012a, ApJL, 750, L12

Del Bene, J. E. 1972, J. Chem. Phys., 57

Di Francesco, J., Hogerheijde, M. R., Welch, W. J., \& Bergin, E. A. 2002, AJ, 124, 2749

Elsila, J. E., Glavin, D. P., \& Dworkin, J. P. 2009, Meteorit. Planet. Sci., 44, 1323
Fedoseev, G., Ioppolo, S., Lamberts, T., et al. 2012, J. Chem. Phys., 137, 54714

Fenimore, C. P., \& Jones, G. W. 1961, J. Phys. Chem., 65, 298

Fujii, N., Chiba, K., Uchida, S., \& Miyama, H. 1986, Chem. Phys. Lett., 127, 141

Garrod, R. T. 2013, Astrophys. J., 765, 60

Garrod, R. T., \& Herbst, E. 2006, Astron. Astrophys., 457, 927

Garrod, R. T., \& Pauly, T. 2011, Astrophys. J., 735, 15

Glavin, D. P., Dworkin, J. P., \& Sandford, S. A. 2008, Meteorit. Planet. Sci., 43, 399

Goicoechea, J. R., Rodríguez-Fernández, N. J., \& Cernicharo, J. 2004, ApJ, 600, 214

Hasegawa, T. I., Herbst, E., \& Leung, C. M. 1992, Astrophys. J. Suppl. Ser., 82, 167

He, J., Frank, P., \& Vidali, G. 2011, Phys. Chem. Chem. Phys., 13,15803

He, J., Jing, D., \& Vidali, G. 2014, Phys. Chem. Chem. Phys., 16, 3493

He, J., \& Vidali, G. 2014, Faraday Discuss., 168

-. 2014, Astrophys. J., 788, 50

Herbst, E., \& van Dishoeck, E. F. 2009, Annu. Rev. Astron. Astrophys., 47, 427

Hidaka, H., Watanabe, M., Kouchi, A., \& Watanabe, N. 2011, Phys. Chem. Chem. Phys., 13, 15798

Hily-Blant, P., Maret, S., Bacmann, A., et al. 2010, A\&A, 521, L.52

Ioppolo, S., Fedoseev, G., Minissale, M., et al. 2014, Physical Chemistry Chemical Physics (Incorporating Faraday Transactions), 16, 8270

Jiménez-Serra, I., Testi, L., Caselli, P., \& Viti, S. 2014, ApJL, 787, L33

Jing, D., He, J., Bonini, M., Brucato, J. R., \& Vidali, G. 2013, J. Phys. Chem. A, 117, 3009

Katz, N., Furman, I., Biham, O., Pirronello, V., \& Vidali, G. 1999, Astrophys. J., 522, 305

Kolasinki, K. W. 2008, Surface science : Foundations of catalysis and nanoscience (Wiley: Hoboken, N.J.)

Le Gal, R., Hily-Blant, P., Faure, A., et al. 2014, A\&A, 562, A83

Nishi, N., Shinohara, H., \& Okuyama, T. 1984, J. Chem. Phys., 80,3898

Perry, R. A. 1984, Chem. Phys. Lett., 106, 223

Pulliam, R. L., McGuire, B. a., \& Remijan, A. J. 2012, Astrophys. J., 751, 1

Sakai, N., Oya, Y., Sakai, T., et al. 2014a, ApJ, 791, L38

Sakai, N., Sakai, T., Hirota, T., et al. 2014b, Nature, 507, 78

Sakai, T., Sakai, N., Foster, J. B., et al. 2013, ApJ, 775, L31

Sanna, A., Cesaroni, R., Moscadelli, L., et al. 2014, A\&A, 565, A34

Shu, J., Lin, J. J., Wang, C. C., Lee, Y. T., \& Yang, X. 2001, J. Chem. Phys., 115, 842

Snow, J. L., Orlova, G., Blagojevic, V., \& Bohme, D. K. 2007, J. Am. Chem. Soc., 129, 9910

Tielens, A. G. G. M. 2005, The Physics and Chemistry of the Interstellar Medium

Turner, B. E. 2000, ApJ, 542, 837

Wang, L., Mebel, A. M., Yang, X., \& Wang, X. 2004, J. Phys. Chem. A, 108, 11644

Wincel, H., Fokkens, R. H., \& Nibbering, N. M. M. 2000, Rapid Commun. Mass Spectrom., 14, 135

Yildiz, U. A., Acharyya, K., Goldsmith, P. F., et al. 2013, 558, A58

Zheng, W., \& Kaiser, R. I. 2010, J. Phys. Chem. A, 114, 5251

Zins, E. L., \& Krim, L. 2014, in 69th International Symposium on Molecular Spectroscopy, in press 